\title{
A novel method for banking stem cells from human exfoliated deciduous teeth: lentiviral TERT immortalization and phenotypical analysis
}

\author{
Zhanhai Yin ${ }^{1 \dagger}$, Qi Wang ${ }^{2 \dagger}$, Ye Li ${ }^{2}$, Hong Wei ${ }^{3}$, Jianfeng Shi ${ }^{3}$ and Ang $\mathrm{Li}^{2,3^{*}}$
}

\begin{abstract}
Background: Stem cells from human exfoliated deciduous teeth (SHED) have recently attracted attention as novel multipotential stem cell sources. However, their application is limited due to in vitro replicative senescence. Ectopic expression of telomerase reverse transcriptase (TERT) is a promising strategy for overcoming this replicative senescence. Nevertheless, its potential application and the phenotype as well as tumorigenicity have never been assessed in SHED.
\end{abstract}

Methods: TERT expression was stably restored in SHED (TERT-SHED) isolated from healthy children aged 6-8 years using lentiviral transduction with a puromycin selection marker. The expression of TERT was detected using reverse transcription polymerase chain reaction, Western blot and immunofluorescence. Surface markers of SHED were detected by flow cytometry. Enzyme-linked immunosorbent assay was used to assess senescence-associated $\beta$-galactosidase, while CCK-8 methods were used to examine the proliferation capacity of SHED and TERT-SHED at different passages. Moreover, multilineage differentiation, karyotype, colony formation in soft agar, and tumor formation in nude mice of SHED and TERT-SHED were also examined.

Results: Lentiviral transduction induced stable TERT expression even in SHED at the 40th passage. TERT-SHED showed robust proliferation capacity and low concentration of $\beta$-galactosidase. Although they had some different biomarkers than early passage SHED, TERT-SHED at late passage showed similar mutilineage differentiation as TERT at early passage. Moreover, TERT-SHED at late passage showed normal karyotype, no soft agar colony formation, and no tumor formation in nude mice.

Conclusions: TERT-immortalized SHED may be a promising resource for stem-cell therapy, although attention should be paid to the biological behavior of the cells.

Keywords: Stem cells from human exfoliated deciduous teeth, Telomerase, TERT, Immortalization, Tumorigenicity

\section{Background}

Tissue engineering depends on the association of stem cells, growth factors, organ tissue culture, and tissue engineering materials [1]. In dentistry, regenerative strategies are of great relevance because of hard dental tissue

\footnotetext{
* Correspondence: drliang234@163.com

${ }^{\dagger}$ Equal contributors

2Department of Periodontology, Stomatological Hospital, College of Medicine, Xi'an Jiaotong University, Xi'an 710004, P. R. China

${ }^{3}$ Research Center for Stomatology, Stomatological Hospital, College of

Medicine, Xi'an Jiaotong University, Xi'an 710004, P. R. China

Full list of author information is available at the end of the article
}

damage, especially as a result of caries lesions, trauma, or iatrogenic procedures. The principles of regenerative medicine can be applied to endodontic tissue engineering. Regeneration of the pulp-dentin complex will allow natural replacement of damaged or missing tooth structures through the activation of tissue-specific stem cells of animal origin or transplantation of stem cells isolated and ex vivo expanded [2]. Stem cells can be found to be quiescent in their niche in all tissues of an adult organism. In response to organ injury, these cells initiate their proliferation and differentiation with the aim of healing 
injured tissue. Among various dental pulp stem cells (DPSCs), stem cells from human exfoliated deciduous teeth (SHED) have recently attracted attention as novel multipotential stem cell sources [3]. The loss of primary teeth creates the perfect opportunity to recover and store this convenient source of stem cells. Isolating SHED is simple, painless and convenient, and involves little or no trauma. These immature stem cells are important in the regeneration and repair of craniofacial defects, tooth loss, and bones because of their capability to proliferate and differentiate [4]. SHED generate rapidly and grow faster than adult stem cells, thus suggesting that they are less mature. SHED are postnatal stem cells capable of differentiating into osteogenic, odontogenic, adipogenic, and neural cells [5].

Although they have high self-renewal capacity, dental stem cells such as SHED will ultimately enter an irreversible proliferation-arrested state referred to as replicative senescence after long term in vitro culture under culture stresses, such as hyperoxia and elevated temperature [6]. Several strategies have been explored to overcome this replicative senescence, such as treatment of SHED with benzopyrene $(\mathrm{BaP})$, radiation, or ectopic expression of viral oncogenes [7]. Long-term treatment with carcinogenic agents such as $\mathrm{BaP}$ results in transformation of cells as evidenced by chromosomal abnormalities, anchorageindependent growth in soft agar, and tumorigenicity in nude mice [8]. Radiation has been shown to be sufficient for immortalization of breast epithelial cells [9]. However, immortalization by radiation occurs relatively infrequently and results in morphological transformation of cells [9] and formation of tumors in nude mice [10]. A number of viral oncogenes, including simian virus-40 (SV40) large $\mathrm{T}$-antigen, adenovirus E1A and E1B, polyoma T-antigen, and papillomavirus E6 and E7, have also been used to immortalize human cells [11-13]. Although immortalized cell lines have been successfully established by transfecting cells with viral oncogenes, inactivation of protein products of the tumor suppressor p53 and retinoblastoma $(\mathrm{Rb})$ $[14,15]$, introduction of karyotypic instability, and transformation of phenotype have been reported in many studies $[16,17]$.

The cellular senescence and the lifespan depend on the loss rate of telomeres during each cell division and the primary length of the telomere [18]. Telomerase reverse transcriptase (TERT; catalysis subunit of telomerase) plays critical roles in the maintenance of telomere length during cell division [19-21]. It has been demonstrated that telomerase reconstitution via TERT expression could extend the telomere, prolong the lifespan of cells, and even immortalize cells $[22,23]$. It has been established that the expression of TERT is a key step in human cellular proliferation, differentiation, and apoptosis. Moreover, recent findings indicate that TERT regulates stem cell properties in stemness sustaining and self-renew characterizations $[24,25]$. While ectopic expression of TERT does significantly lengthen the lifespan of cells, enhanced telomerase activity is also a feature of many types of tumors and malignancies $[26,27]$. The potential tumorigenicity of TERT-expressed stem cells remains controversial [27-29]. Therefore, the tumorigenicity of TERT expression in human stem cells needs to be further validated.

Our primary goal in this study was to create an immortalized SHED cell line by stable expression of TERT. Moreover, we assessed the multipotency and the potential tumorigenicity of our immortalized SHED cell line.

\section{Methods}

\section{Subjects and cell culture}

The SHED were obtained from the deciduous teeth of children aged 6-8 years. Every patient involved in the study consented to participate in the study and signed the paper consent. This study was approved according to guidelines set by the Ethic Committee of the Dental Hospital, Xi'an Jiaotong University. The deciduous anterior teeth used in this study were near natural exfoliation, with less than one third of the root remaining, and without any deep caries, restoration, periapical lesions, or internal resorption. After extraction, pulp tissues from the deciduous teeth were extirpated using a barbed broach (Mani, Utsunomiya Toshi-ken, Japan), washed with phosphate-buffered saline (PBS; Invitrogen, Carlsbad, CA, USA), and then treated with collagenase type I ( $3 \mathrm{mg} / \mathrm{ml}$; Invitrogen) and dispase $(4 \mathrm{mg} / \mathrm{ml}$; Invitrogen) for $30 \mathrm{~min}$ at $37^{\circ} \mathrm{C}$; they were then filtered through a 70$\mu \mathrm{m}$ cell strainer. The SHED were cultured in a DMEM/ F12 medium supplemented with $15 \%$ fetal bovine serum, $2 \mathrm{mmol} / \mathrm{l} \mathrm{L}$-glutamine, $100 \mu \mathrm{mol} / \mathrm{l} \mathrm{L}$-ascorbic acid-2phosphate, $100 \mathrm{U} / \mathrm{ml}$ penicillin, $100 \mu \mathrm{g} / \mathrm{ml}$ streptomycin, and $0.25 \mu \mathrm{g} / \mathrm{ml}$ amphotericin B. After 7 days, cell colonies were observed. Individual cell colonies were collected by the filter paper enzyme digestion method. The derived cells were SHED.

\section{Cloning of TERT in lentiviral expression plasmid and lentiviral production}

pCMV6-XL5 plasmid (OriGene Technologies, Beijing, China) containing full-length cDNA of human TERT (3.6 kb) [Genbank:NM_198253.2] was amplified in DH5a E. coli strain. The cDNA clone of TERT and GV166 lentiviral vector (GeneChem Co., Ltd., Shanghai, China) were digested by a cocktail of EcoR I and Sal I (New England Biolabs, Ipswich, USA). The subsequent fragments were purified and recombined by T4 ligase (New England Biolabs) and then transformed into DH5 $\alpha$ E. coli selecting for ampicillin resistance. The transformants were screened for correct insertion/orientation of the TERT fragment by restriction analysis. GV166 vector not recombined with 
TERT was used as the control vector. For lentiviral production, the GV166-TERT or control plasmid was co-transfected into 293FT cells with Lenti-Easy Packaging Mix (GeneChem Co., Ltd.) at a 1:3 ratio using Lipofectamine $^{\mathrm{Tu}}$ reagent (Invitrogen). Forty-eight hours after transfection, the virus-containing supernatant was harvested and stored in aliquots at $-80{ }^{\circ} \mathrm{C}$. All cell culture procedures were performed under biosafety level 2 conditions.

\section{Transduction of SHED with lentiviral vectors}

Cells were plated $24 \mathrm{~h}$ before transduction at a density of $5 \times 10^{4}$ cells per well in six-well plates in the presence of $5 \mu \mathrm{g} / \mathrm{ml}$ polybrene. Transduction of SHED was carried out with TERT or control lentivirus at a multiplicity of infection (MOI) of 65. Transduced cells were passaged, and selected with puromycin $(1.5 \mathrm{mg} / \mathrm{ml})$ for 5 days.

\section{Extraction of total RNA and RT-PCR}

Total RNA was extracted using TRIzol reagent (Invitrogen, Carlsbad, CA, USA) according to the manufacturer's protocol, and RNase-free DNase I was used to remove DNA contamination. Reverse transcription (RT) was performed with $2 \mu \mathrm{g}$ total RNA using M-MLV Reverse transcriptase (Promega, Madison, WI, USA) to synthesize first-strand cDNA according to the manufacturer's recommendation, followed by cDNA amplification using the specific primers for TERT and the $\beta$-actin primer. Primers used in this study were as follows: 5 '-AGAGTGTCTGGAGCAAGTTG-3' (forward) and 5'-GGATGAAGCGGAGTCTGG-3' (reverse) for TERT; $5^{\prime}$-ATCGTGCGTGACATTAAGGAG AAG-3' (forward) and 5'-GAGGAAGGAAGGCTGG AAGAGTG-3' (reverse) for $\beta$-actin; and the corresponding polymerase chain reaction (PCR) products were $140 \mathrm{bp}$ and $179 \mathrm{bp}$, respectively.

\section{Western blot}

Cells were lysed in RIPA lysis buffer, and the lysates were harvested by centrifugation $(13,523 \times \mathrm{g})$ at $4{ }^{\circ} \mathrm{C}$ for $30 \mathrm{~min}$. Approximately $20 \mu \mathrm{g}$ protein samples were then separated by electrophoresis in a $12 \%$ sodium dodecyl sulfate polyacrylamide gel and transferred onto a polyvinylidene fluoride membrane. After blocking the nonspecific binding sites for 60 min with $5 \%$ non-fat milk, the membranes were incubated overnight at $4{ }^{\circ} \mathrm{C}$ with a mouse monoclonal antibody against human TERT (Abgent, USA, at a 1:1000 dilution). The membranes were then washed three times with TBST (tris-buffered saline with tween-20) for $10 \mathrm{~min}$ and probed with the horseradish peroxidase (HRP)-conjugated rabbit antimouse IgG antibody (Immunology Consultants Laboratory, USA, at a $1: 2000$ dilution) at $37{ }^{\circ} \mathrm{C}$ for $1 \mathrm{~h}$. After three washes, the membranes were developed by an enhanced chemiluminescence system (Cell Signaling
Technology, Danvers, MA, USA). A rabbit polyclonal antibody against human $\beta$-actin (Abcam, UK, at a 1:10000) was set as the inner control.

\section{Immunofluorescence staining}

Cells were fixed in $4 \%$ paraformaldehyde, permeabilized with $0.1 \%$ Triton1-X100, and blocked with $10 \%$ horse serum in PBS for $1 \mathrm{~h}$. The TERT antibody was prepared at 1:100 dilution and further incubated with the samples for $18 \mathrm{~h}$ at $4{ }^{\circ} \mathrm{C}$. After washing with PBS, the cell was incubated with FITC-labeled goat antimouse antibody (Invitrogen) at 1:500 dilution for $45 \mathrm{~min}$. Cells were also counterstained with DAPI. The fluorescence was evaluated by fluorescence microscope (Apotome).

\section{Detection of surface markers by flow cytometry}

The stem cell nature of SHED was analyzed using flow cytometry. Cells cultured with basal medium before cell differentiation were harvested using trypsin and washed twice with PBS. For cell surface staining, cells were fixed, washed and incubated with FITC-conjugated monoclonal or polyclonal antibodies against CD34, CD45, CD146 or STRO-1 (all Biolegend, CA, USA). For intracellular staining, cells were fixed and permeabilized using the Fix \& Perm kit (Invitrogen), then washed and incubated with FITC-conjugated monoclonal or polyclonal antibodies against Oct-4 or Nanog (both Biolegend). FITC-conjugated IgG (Biolegend) was used as a negative control. The cells were analyzed using a FACScan flow cytometer (Becton Dickinson, Franklin Lakes, NJ, USA).

\section{Multilineage differentiation assays}

In vitro osteogenic differentiation of SHED was performed as previously published [30] using $100 \mathrm{nM}$ dexamethasone, $10 \mathrm{mM} \beta$-glycerophosphate, and $50 \mathrm{M} \mathrm{L}$-ascorbic acid-2-phosphate (Sigma). A total of $5 \times 10^{3}$ cells/well were seeded on to a six-well plate. After 16 days cells were assayed by von Kossa staining using a standard protocol. Adipogenic differentiation was accomplished as previously published [30] by $1 \mathrm{M}$ dexamethasone, $0.2 \mathrm{mM}$ indomethacin, $0.1 \mathrm{mg} / \mathrm{ml}$ insulin, and $1 \mathrm{mM}$ 3-isobutyl-1-methylxanthin (IBMX) (Sigma). The maintenance medium consisted of $0.1 \mathrm{mg} / \mathrm{ml}$ insulin in standard medium. A total of $4 \times 10^{3}$ cells/well were seeded on to a 12-well plate. Stimulation was started when cells reached full confluency. Cells were grown for 5 days in induction medium and thereafter for 2 days in maintenance medium, and were then switched to induction medium again. After 16 days of stimulation, the cells were assayed by oil red staining using a standard protocol. Chondrogenic differentiation was achieved in aggregate cultures as previously published [30] with $100 \mathrm{nM}$ dexamethasone, $1 \mathrm{mM}$ pyruvate, $195 \mathrm{M}$ L-ascorbic acid-2-phosphate, 350 M L-proline, $1.25 \%$ (v/v) insulin-transferrin-selenious 
acid mix (ITS, 100×), $5.35 \mathrm{~g} / \mathrm{ml}$ linolic acid, $1.25 \mathrm{mg} / \mathrm{ml}$ bovine serum albumin (BSA; Sigma), and transforming growth factor-3 (TGF-3, $10 \mathrm{ng} / \mathrm{ml}$; R\&D Systems, Minneapolis, MN, USA). A total of $2.5 \times 10^{5}$ cells were used per pellet. Sections $(12 \mu \mathrm{m})$ were cut with a cryostat vacutome HM 200 OM (Microm, Walldorf, Germany). Anionic sulfated proteoglycans were detected by toluidine blue metachromasia. Slices were stained in $1 \%$ toluidine blue solution (Sigma, Munich, Germany) and $1 \%$ sodium tetraborate (Sigma).

\section{Real-time PCR}

Total RNA and cDNA of differentiation-induced cells were prepared according to the above-mentioned protocols. Differentiation markers and GAPDH were amplified by quantitative real-time PCR using the following primers: $A L P$ forward 5' -CATGCTGAGTGACACAGACAAGAA-3', reverse: 5'-ACAGCAGACTGCGCCTGGTA-3'; $B S P$ forward 5'-CTGGCACAGGGTATACAGGGTTAG-3', reverse: 5'-GCCTCTGTGCTGTTGGTACTGGT-3'; LPL forward: 5' -GTCACGGGCTCAGGAGCATTA-3', reverse: 5'-GCTCCAAGGCTGTATCCCAAGA-3'; $P P A \gamma-2$ forward: 5' -GCTCTGCAGGAGATCACAGA-3', reverse: 5' -GGGCTCCATAAAGTCACCAA-3'; ACAN forward: 5' -ACGAAGACGGCTTCCACCAG-3', reverse: 5'-TCGG ATGCCATACGTCCTCA-3'; COL2A1 forward: 5'-CCAG TTGGGAGTAATGCAAGGA-3', reverse: $5^{\prime}$-ACACCAG GTTCACCAGGTTCA-3'; GAPDH forward: 5'-CTCCTC CTGTTCGACAGTCAGC-3', reverse: $5^{\prime}$-CCCAATACGA CCAAATCCGTT-3'. Gene-specific amplification was performed in an ABI 7900HT real-time PCR system (Life Technologies, Carlsbad, CA) with a $15-\mu$ l PCR mix containing $0.5 \mu \mathrm{l}$ cDNA, $7.5 \mu \mathrm{l} 2 \times$ SYBR Green master mix (Invitrogen), and $200 \mathrm{nM}$ of the appropriate primers. The mix was preheated at $95{ }^{\circ} \mathrm{C}$ for $10 \mathrm{~min}$ and then amplified in 45 cycles of $95^{\circ} \mathrm{C}$ for $30 \mathrm{~s}$ and $60^{\circ} \mathrm{C}$ for $1 \mathrm{~min}$. The resolution curve was measured at $95{ }^{\circ} \mathrm{C}$ for $15 \mathrm{~s}, 60^{\circ} \mathrm{C}$ for $15 \mathrm{~s}$, and $95{ }^{\circ} \mathrm{C}$ for $15 \mathrm{~s}$. The $\mathrm{Ct}$ (threshold cycle) value of each sample was calculated, and the relative mRNA expression was normalized to the GAPDH value $\left(2^{-\Delta \Delta C t}\right.$ method). The final expression value of differentiation markers was standardized according to that of control cultures.

\section{Senescence-associated $\beta$-galactosidase assay by ELISA}

Cells $\left(1 \times 10^{6}\right)$ were lysed and the supernatant was collected by centrifuge. The activity of $\beta$-galactosidase ( $\beta$-GAL) in SHED was assessed using the human $\beta$-GAL enzyme-linked immunosorbent assay (ELISA) Kit (CSBE09463h, Cusabio, China) according to the manufacturer's recommendations.

\section{Proliferation assay}

Cells were plated at a density of $1 \times 10^{3} /$ well in 96-well plates and cultured in basal medium. A CCK- 8 assay was performed twice a day according to the cell counting kit protocol (Keygen Biotech, Nanjing, China) for 12 consecutive days. The values for each well were spectrophotometrically measured at $450 \mathrm{~nm}$.

\section{Cytogenetic analysis}

Metaphase spreads were prepared from exponentially growing TERT-SHED at various passages. Cells were harvested and fixed following standard protocols [31]. Chromosome analysis was performed using the GTGbanding technique [31]. Fifteen metaphases captured by a CCD camera were analyzed and karyotyped using the CytoVision system (Leica Biosystems, Nussloch, Germany). Chromosome identification and karyotype description were made in accordance with the International System for Chromosome Nomenclature [32].

\section{DNA isolation and PCR analysis of CDKN2A}

Genomic DNA from SHED P4, and TERT-SHED P20 and $\mathrm{P} 40$ was extracted using the QIAamp DNA Mini Kit (QIAGEN, Duesseldorf, Germany) according to the manufacturer's recommendations. The exon 2 of the $C D K N 2 A$ gene was amplified in an ABI GeneAmp 9600 PCR system using the following primers: forward: $5^{\prime}-\mathrm{CC}$ TGGCTCTGACCATTCTGTTC-3', reverse: 5'-GCTTTG GAAGCTCTCAGGGTAC-3'. The PCR mixture contained 100 ng genomic DNA, $10 \mu \mathrm{l} 2 \times$ Taq PCR Master Mix (TIANGEN Biotech, Beijing, China), and $0.1 \mathrm{pmol} / \mu \mathrm{l}$ of each primer in a total $20 \mu \mathrm{l}$ volume. The PCR cycling conditions were $94{ }^{\circ} \mathrm{C}(2 \mathrm{~min})$ for $1 \mathrm{cycle}, 94{ }^{\circ} \mathrm{C}(30 \mathrm{~s}), 55{ }^{\circ} \mathrm{C}$ (40 s), $72{ }^{\circ} \mathrm{C}(2 \mathrm{~min})$ for 36 cycles, and a final extension of $72{ }^{\circ} \mathrm{C}$ (10 min). The PCR products were $386 \mathrm{bp}$ in length.

\section{Soft agar assay}

TERT-SHED or tongue cancer cells (Tca-8113; 2500 cells) in logarithmic growth phase were trypsinized and suspended into a single cell suspension in $0.5 \mathrm{ml} 0.8 \%$ top agar solution $\left(37^{\circ} \mathrm{C}\right)$. The cells were aliquoted on the top of a pre-prepared $1 \mathrm{ml} 1.2 \%$ base agar layer, and then incubated for 28 days at $37{ }^{\circ} \mathrm{C}$ with $5 \% \mathrm{CO}_{2}$. The well plates were stained with $0.005 \%$ crystal violet for $2 \mathrm{~h}$ before a photograph was taken under a microscope [33].

\section{Tumorigenicity in nude mice}

Twenty athymic nude mice were divided into four groups: SHED P2, TERT-SHED P20, Tca-8113, and PBS control. Cells $\left(2 \times 10^{6}\right)$ of each cell type were suspended in $200 \mu \mathrm{l}$ PBS and injected subcutaneously into the fore and hind limb armpit under general anesthesia. Mice were sacrificed after 8 weeks by $\mathrm{CO}_{2}$ overdose. All procedures were performed according to animal protection legislation and approved by the Ethics Committee of the Dental Hospital, Xi'an Jiaotong University. Photographs were taken every week for macroscopic evaluation. Skin 
and underlying soft tissue of the relevant area were dissected 8 weeks after cell implantation. Slides were prepared and stained with hematoxylin and eosin dye, and investigated for possible tumor growth [34].

\section{Statistical analysis}

The data for $\beta$-GAL concentration and proliferation were expressed as mean \pm standard deviation (SD). Oneway proliferation data were used to compare the difference in $\beta$-GAL and followed by Fisher's LSD post hoc test. Repeated measurement analysis of variance was used to compare the differences between proliferation curves. All statistical analyses were performed using IBM SPSS Statistics 19.0 software and a $P$ value $<0.05$ was considered significant.

\section{Results}

\section{SHED morphology}

The morphology of SHED was analyzed under phase contrast microscope (Fig. 1). SHED spread along the surface of the culture plates, showing rapid growth within the first week. Cell colonies were seen from the fifth day, as the cells grew to confluence at 10-14 days.

\section{Expression of TERT in SHED after lentiviral transduction}

We first analyzed the expression of TERT in SHED after stable lentiviral transduction using Western blot and immunofluorescence. As shown in Fig. 2, TERT transduction remarkably restored the expression of TERT in SHED (TERT-SHED), while the control virus did not show TERT expression (SHED).

\section{Expression of TERT in TERT-SHED during passage}

We further analyzed the expression of TERT during the passage of TERT-SHED using RT-PCR and Western blot.

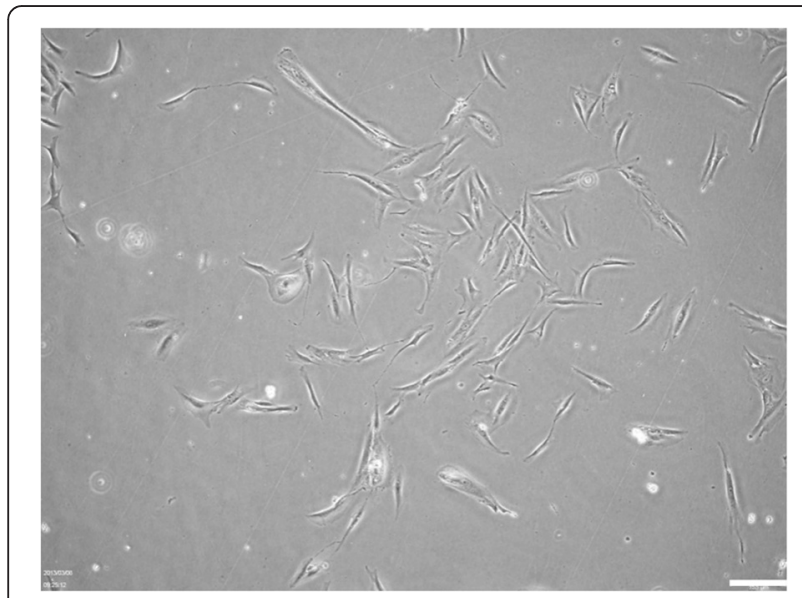

Fig. 1 Typical cell morphology of SHED under phase contrast microscope. SHED revealed a typical fibroblast-like morphology. scale bar $=50 \mu \mathrm{m}$
As shown in Fig. 3, TERT expression remained stable even in the 40th passage.

\section{Surface markers of SHED}

The flow cytometry analysis was applied to quantify the expression ratios of specific surface antigens in SHED and TERT-SHED. The SHED at the fourth passage (SHED P4) showed robust expression of CD146 (90.45\%), STRO-1 (72.10 \%), CD34 (65.76\%), and Oct-4 (85.16\%), weak expression of Nanog (13.84\%), and nearly negative expression of CD45 (3.79 \%) (Fig. 4a). The TERT-SHED at the 20th passage (SHED P20) showed decreased expression of CD146 (48.51 \%), STRO-1 (58.47 \%), Oct-4 (10.48\%), Nanog (7.64 \%), and CD34 (3.71 \%) (Fig. 4b).

\section{Multilineage differentiation of SHED}

The multilineage differentiation potential of SHED was measured by differentiation induction assay. SHED P4 could different into osteogenic, adipogenic, and chondral cell lineages, as revealed by positive staining for Alizarin Red S, Oil Red $\mathrm{O}$ and Toluidine blue, respectively (Fig. 5a-c). Moreover, TERT-SHED P20 showed similar differentiation capacity to SHED P4 (Fig. 5e-g). Furthermore, real-time PCR analysis confirmed that TERT-SHED P20 and SHED P4 had similar expression of osteogenic (ALP and BSP), adipogenic (LPL and PPAR- $\gamma$ ), and chondrogenic (ACAN and COL2A1) differentiation markers (Fig. 5d and h).

\section{Cell senescence and proliferation capacity of SHED}

$\beta$-GAL activity at pH 6 is a known characteristic of senescent cells which is not found in presenescent, quiescent or immortal cells. We first examined the senescence marker, $\beta$-GAL, in SHED and TERT-SHED at different passages. As shown in Fig. $6 a$, the concentration of $\beta$-GAL in the 20th passage of SHED (SHED P20) was as 120 times that in SHED P4. However, $\beta$-GAL concentration remained at a very low level in the 40th passage of TERT-SHED (TERT-SHED P40). The proliferation capacity of SHED was detected by CCK- 8 assay. As shown in Fig. 6b, the proliferation capacity of SHED at late passage (P20) significantly decreased. However, TERT-SHED P40 had similar proliferation potential to the early passage (P4) of SHED.

\section{Cytogenetic of TERT-SHED}

Atypia is one of the major characteristics of cancer. We therefore analyzed the karyotype of TERT-SHED at late passage (P20). As shown in Fig. 7a, no polyploid mutation or chromosomal deletion was found in TERT-SHED P40. 


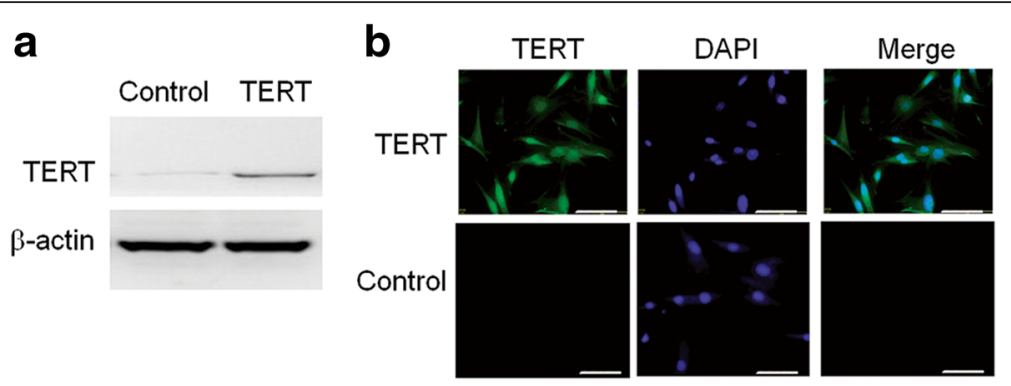

Fig. 2 Expression of TERT assessed by Western blot (a) and immunofluorescence (b) in SHED at the 20th passage $60 \mathrm{~h}$ after control and lentiviral transduction. Transduction of TERT-recombined lentivirus restored the expression of TERT in SHED and the optimal MOI was 65 . Scale bar $=50 \mu \mathrm{m}$. TERT telomerase reverse transcriptase

\section{Integrity of genomic CDKN2A}

Since a deletion of the CDKN2A gene locus has been described after ectopic TERT expression using retroviral vectors [35], we analyzed the integrity of $C D K N 2 A$. PCR amplification yielded a band of the expected size for SHED P4, TERT-SHED P20 and TERT-SHED P40 (Fig. 7b).

\section{Colony formation of TERT-SHED}

The soft agar colony formation assay is a common method to monitor anchorage-independent growth. We thus examined the colony formation of TERT-SHED P20. As shown in Fig. 7c, only a single cell was noticed growing in soft agar in the culture of TERT-SHED P20, while cell aggregates were formed in the culture of tongue cancer cells (Tca-8113).

\section{Tumorigenicity of TERT-SHED in nude mice}

We further assessed tumor formation of TERT-SHED P20 in nude mice. As shown in Fig. 7d, no tumor formation was seen in TERT-SHED P20. However, tumor formation was noticed after Tca-8113 cell inoculation.

\section{Discussion}

In this study, we established a method to immortalize SHED using ectopic stable expression of TERT by lentiviral vector. We found that TERT-SHED showed a robust proliferation capacity even in late passages without cell senescence as indicated by low activity of $\beta$-GAL. Although they had some different biomarkers compared to early-passage SHED, TERT-SHED at late passage showed similar mutilineage differentiation to TERT at early passage. We also assessed the potential tumorigenicity of TERTSHED, and found that TERT-SHED at late passage showed low tumorigenicity, as indicated by normal karyotype, no soft agar colony formation, and no tumor formation in nude mice. These data suggest that TERT expression may be a safe technique for banking SHED for tissue repair.

SHED are mesenchymal-like cells and are an attractive candidate for use in tissue repair thanks to their multipotentiality, easy availability, and immunoprivileged status [36]. They do not induce an allogenic reaction and may even suppress host T-cell proliferation [37], suggesting that cells cultured from a single donor may be expanded in vitro to form a reserve pool that could be used for multiple recipients. However, during in vitro culturing, SHED undergoes replicative senescence and

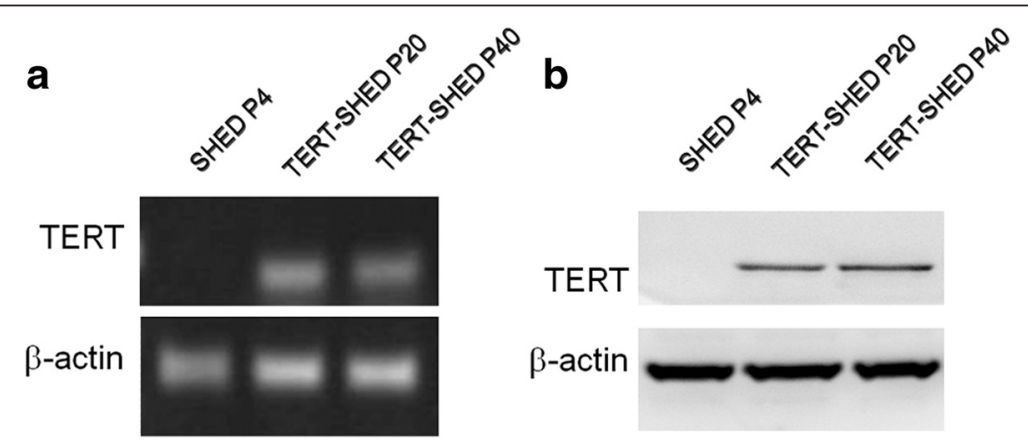

Fig. 3 Expression of TERT assessed by RT-PCR (a) and Western blot (b) in SHED and TERT-SHED at different passages. SHED showed robust mRNA and protein expression of TERT even at the 40th passage after lentiviral transduction. $P$ passage, SHED stem cells from human exfoliated deciduous teeth, TERT telomerase reverse transcriptase 

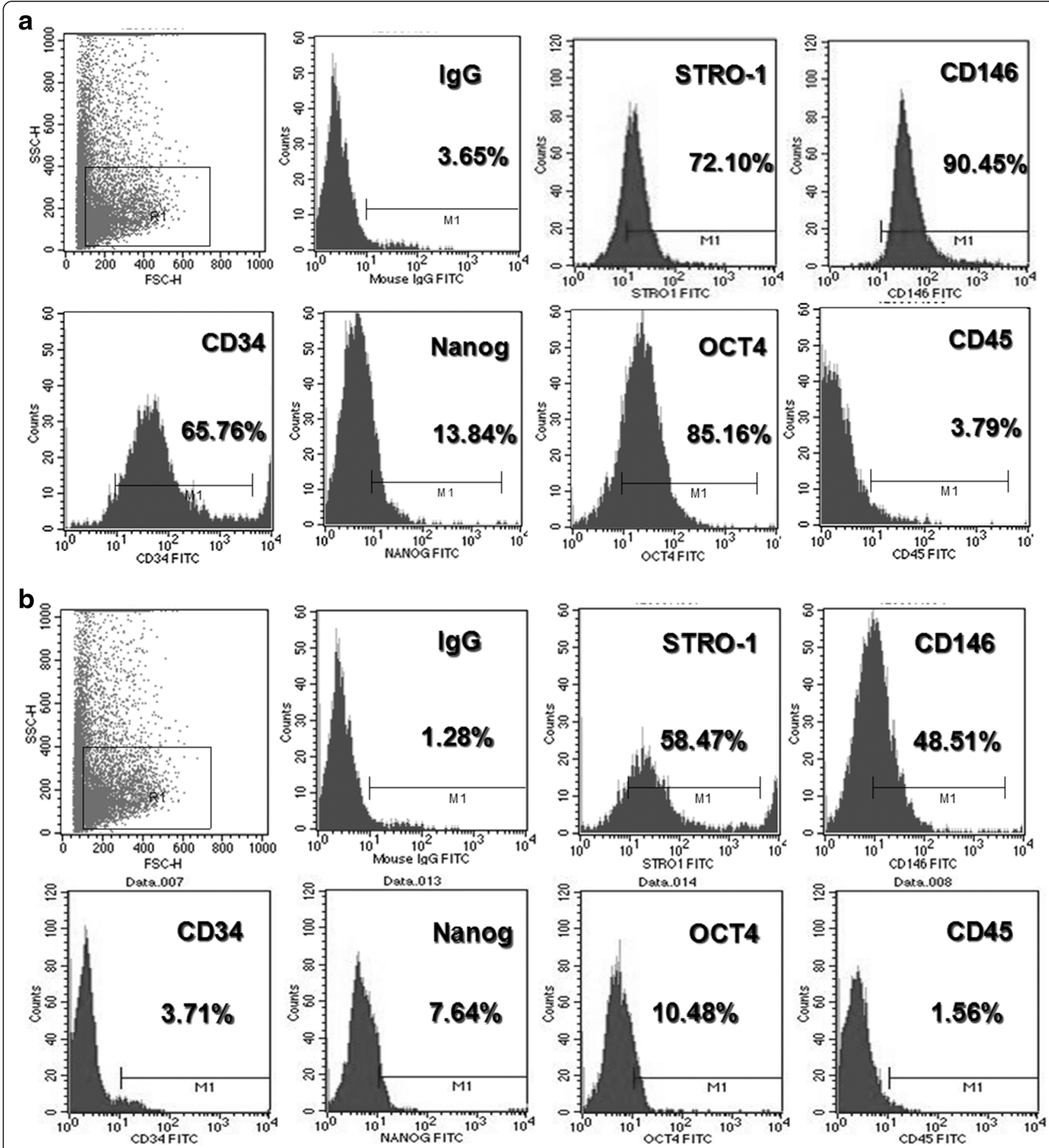

Fig. 4 Surface marker of SHED P4 (a) and TERT-SHED P2O (b) as assessed by flow cytometry. TERT-SHED retained the expression of stem cell markers, such as STRO-1, CD146, and Nanog, at the 20th passage. However, the expression of CD34 and OCT4 was downregulated at the 20th passage, whereas the expression of CD45 remained at a low level

loses its ability to differentiate over time [38]. Thus, immortalization of dental stem cells (DSCs) and establishment of a dental stem cell line are important for DSC research and regenerative dentistry.

It is generally thought that replicative senescence of stem cells is a result of genetic instability after critical shortening of telomeres [39]. Telomerase had the enzymatic activity to maintain and elongate telomere length during cell division. Ectopic expression of TERT has been proven in many studies to maintain the telomere length in different types of cells, thus immortalize cells and prevent cells from loss of function. Using this approach, stable 


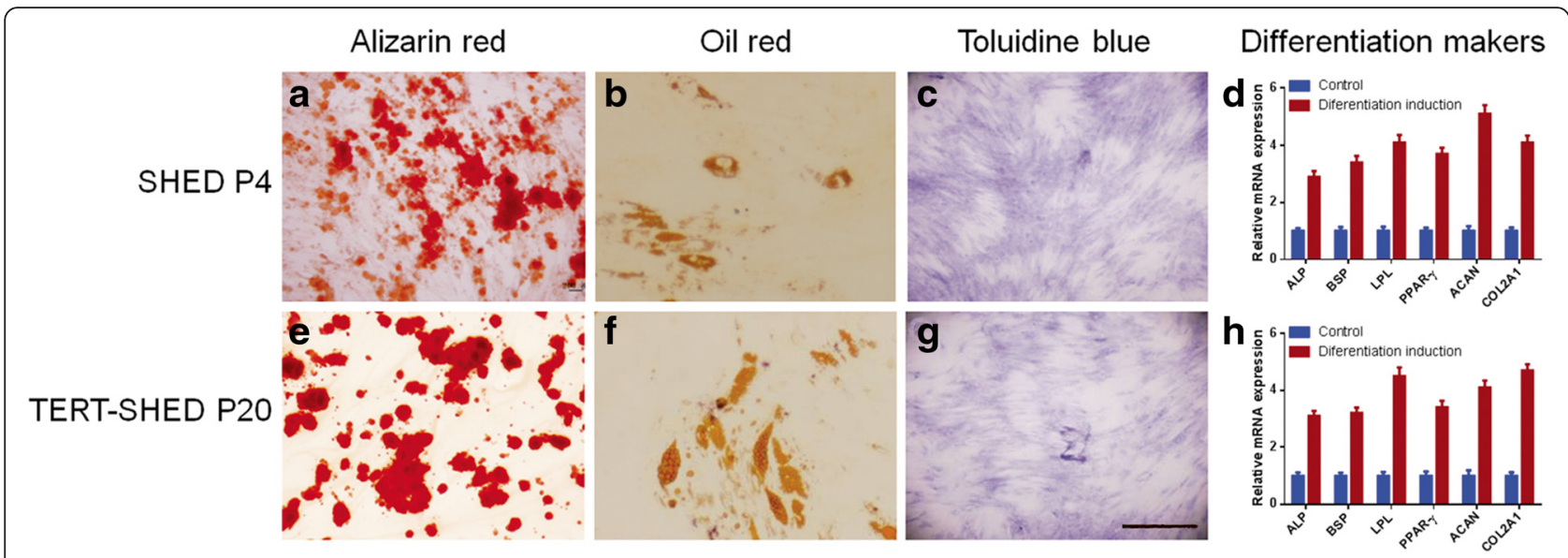

Fig. 5 Multilineage differentiation assay of SHED P4 and TERT-SHED P20. Calcium deposition around cells was stained red by Alizarin Red S (a and $\mathbf{e}$ ) after induction; adipose droplets in cells were stained orange by Oil Red $\mathrm{O}$ after adipogenic induction (b and $\mathbf{f}$ ); proteoglycans in cells were stained blue by Toluidine blue after chondrogenic induction ( $\mathbf{c}$ and $\mathbf{g}$ ). The expression of osteogenic, adipogenic, and chondrogenic differentiation markers were examined using real-time PCR and standardized according to that of control culture (d and $\mathbf{h}$ ). TERT-SHED P20 showed similar osteogenic, adipogenic, and chondrogenic differentiation to SHED P4. Scale bar $=50 \mu \mathrm{m}$. P passage, SHED stem cells from human exfoliated deciduous teeth, TERT telomerase reverse transcriptase

expression of TERT prevents replicative senescence in human mesenchymal stem cells (hMSCs) [40-43], with a lifespan extension of more than 3 years [44]. These findings are consistent with our results that stable TERT expression causes a continuous proliferation of SHED with a lack of senescence-associated $\beta$-GAL staining in robust cells even in the 40th passage, whereas untransduced cells went into senescence in the 20th passage, indicating that TERT expression may be a useful strategy for immortalizing stem cells.

Usually, long-term in vitro culture of stem cells results in impaired differentiation capacity [45]. Previous studies have demonstrated that MSCs overexpressing TERT exhibit an increased osteogenic differentiation potential [45], while telomerase deficiency impairs differentiation of hMSCs [46]. In our study, although TERT-SHED at late passage showed some decrease in the biomarkers, its differentiation into osteal, adipic, and chondric cells was similar to that in SHED at early passage, suggesting that telomere length maintenance plays an important role in the differentiation of stem cells.

With the success of immortalization of stem cells by TERT, concerns about potential malignant transformation by viral TERT transduction were raised. It has been reported that long-term culture of TERT-transduced adult MSCs using a retrovirus resulted in neoplastic transformation

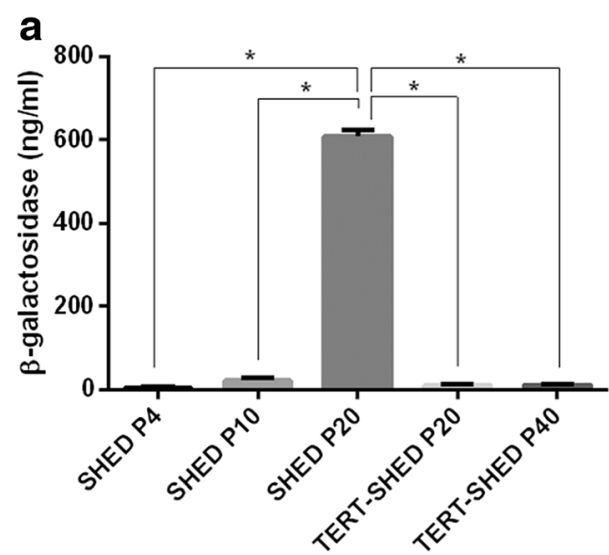

b

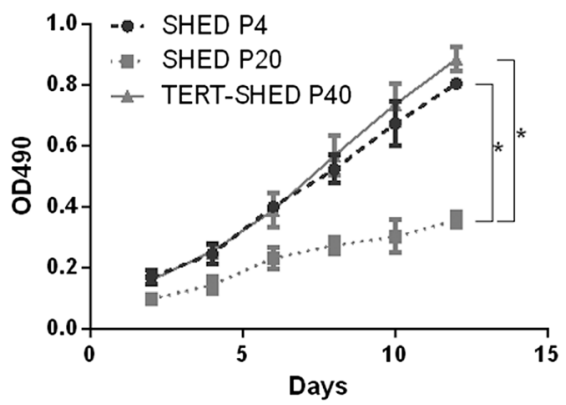

Fig. 6 Comparison of $\beta$-GAL expression and proliferation capacity of SHED and TERT-SHED at different passages. a TERT-SHED showed low $\beta$-GAL expression at a late passage (40th passage; P40), whereas SHED P20 showed senescence as indicated by remarkably high $\beta$-GAL expression. $\mathbf{b}$ TERT-SHED at late passages (20th and 40th passages; P2O and P40) showed a significantly stronger capacity for proliferation than SHED at the fourth passage (P4). ${ }^{*} P<0.05$. OD optical density, SHED stem cells from human exfoliated deciduous teeth, TERT telomerase reverse transcriptase 


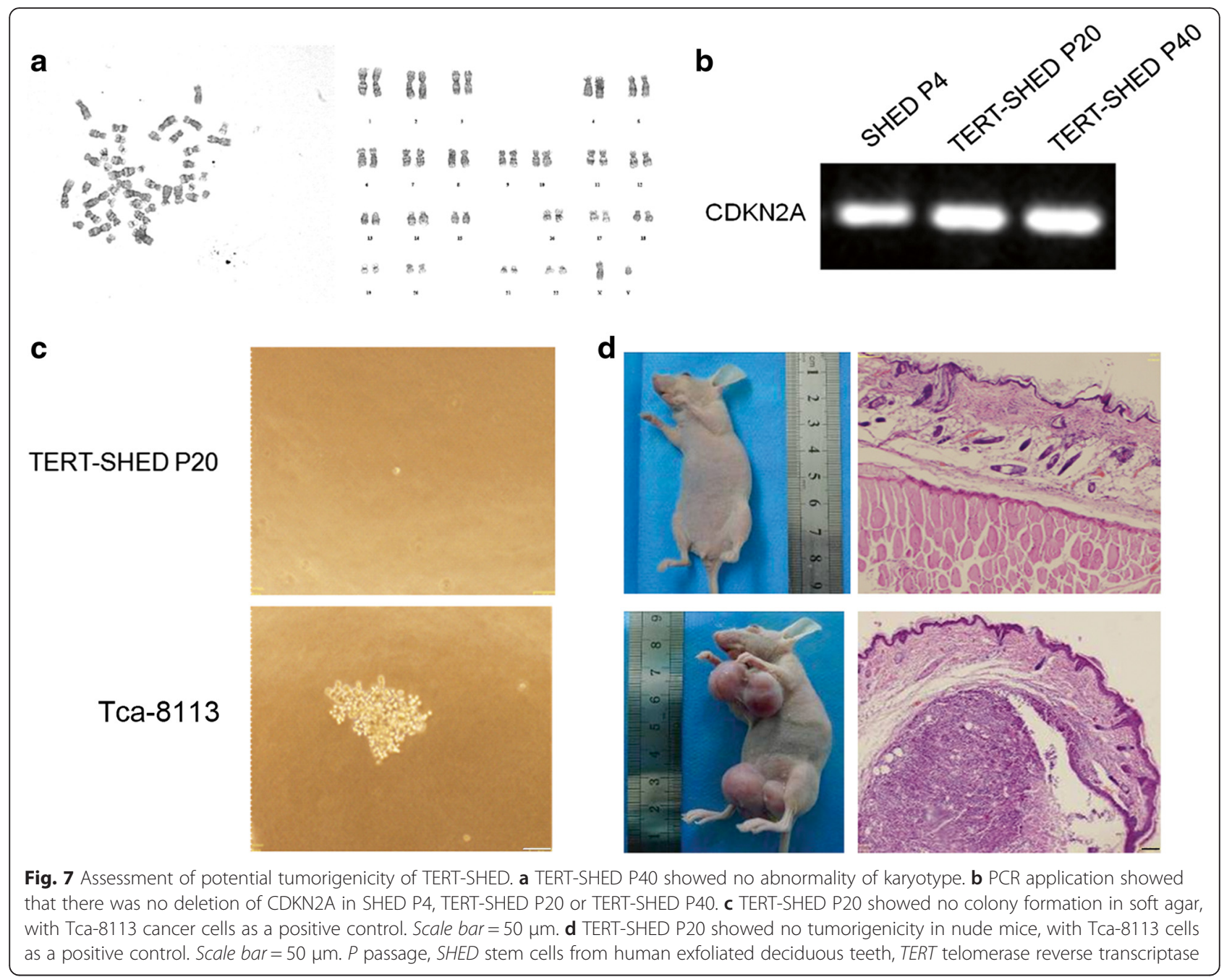

[26, 35]. Insertional mutagenesis by long terminal repeat (LTR) has been a limitation of retroviral gene transfer [35] since oncogenesis occurred at an unexpected high frequency in the X-SCID gene therapy trial. However, so far, all available data suggest that lentiviral vectors are safe vehicles for ex vivo gene therapy and no adverse events have been reported upon transplantation of lentivirus vector-transduced cells [47]. The major reason for the low genotoxicity of lentiviral vectors may be the lack of transcriptionally active LTR. On the other hand, telomere length maintenance plays critical roles in preventing chromosomal instability and subsequent carcinogenesis. Markedly elevated risks of tumors (about 11 times that of the general population) are observed in patients with dyskeratosis congenita, a disease with very short telomeres caused by germline mutations in the components of the telomerase complex [18]. Mouse models also support the notion that abnormally short telomere length increases the risk of cancers [48]. Recent prospective epidemiological studies have demonstrated that a short telomere is significantly associated with increased cancer incidence and death $[49,50]$. Therefore, it is reasonable that lentiviral vector-mediated TERT expression had low tumorigenicity in SHED at late passage, as indicated by no abnormal karyotype, no colony formation in soft agar, and no tumor formation in nude mice. However, our findings need to be extended in SHED over hundreds of passages, and emphasize the caution in the use of TERT-immortalized cells in studies of normal cell biology and in tissue engineering.

There are several limitations in our study. Like most laboratory studies [5], our SHED were maintained and expanded in bovine serum-containing medium, which raises the concern about its clinical application due to the high lot-to-lot variability, risk of contamination, and immune response against xenogenic proteins in bovine serum [51]. Because of the numerous constituents of bovine serum, the development of chemically defined serum-free media with an optimal composition of the few essential factors is only beginning. Thus, bovine 
serum remains the gold standard medium supplement for laboratory-scale MSC culture and has been used in clinical trials approved by the US Food and Drug Administration [52]. Recent studies have demonstrated that human blood-derived components may be an ideal substitute for bovine serum in the therapeutic application of stem cells. Therefore, SHED expanded in xeno-free media are needed for clinical therapy.

\section{Conclusions}

In this study, we show that a lentiviral TERT gene transduction could establish a stable SHED cell line that is completely multipotential; even after long-term in vitro passaging, no evidence of genetic instability or malignant biological behavior of these cells was observed. These findings provide novel strategies to prevent the senescence and maintain the stemness of ex vivo-maintained SHED for potential clinical therapies, although attention should be paid to the biological behavior of these cells.

\section{Abbreviations \\ BaP: benzopyrene; $\beta$-GAL: $\beta$-galatosidase; Ct: threshold cycle; DSC: dental stem cell; ELISA: enzyme-linked immunosorbent assay; hMSC: human mesenchymal stem cell; LTR: long terminal repeat; MOI: multiplicity of infection; MSC: mesenchymal stem cell; P: passage; PBS: phosphate-buffered saline; PCR: polymerase chain reaction; RT: reverse transcription; SHED: stem cells from human exfoliated deciduous teeth; TERT: telomerase reverse transcriptase.}

\section{Competing interests}

The authors declare that they have no competing interests.

\section{Authors' contributions}

$A L$ and ZY designed the study. QW and YL were responsible for collection and/or assembly of experimental data. HW and JS were responsible for conception and design, data analysis and interpretation. AL and ZY drafted the manuscript and revised the manuscript. All authors read and approved the final manuscript.

\section{Acknowledgments}

This study was supported by grants from the Shaanxi Provincial Science and Technology Innovation Project co-ordination of the Resources Oriented Industries of Key Technologies Project (2011KTCL03-24), Shaanxi Province Natural Science Basic Research (2013JM4042), Fundamental Research Funds for the Central Universities of China, and National Natural Science Foundation of China $(30801173,81371943)$.

\section{Author details}

'Department of Orthopedics, First Affiliated Hospital, College of Medicine, Xi'an Jiaotong University, Xi'an 710061, P. R. China. ${ }^{2}$ Department of Periodontology, Stomatological Hospital, College of Medicine, Xi'an Jiaotong University, Xi'an 710004, P. R. China. ${ }^{3}$ Research Center for Stomatology, Stomatological Hospital, College of Medicine, Xi'an Jiaotong University, Xi'an 710004, P. R. China.

Received: 6 August 2015 Revised: 31 January 2016

Accepted: 21 March 2016

Published online: 04 April 2016

\section{References}

1. Murray PE, Garcia-Godoy F, Hargreaves KM. Regenerative endodontics: a review of current status and a call for action. J Endod. 2007;33(4):377-90. doi:10.1016/j.joen.2006.09.013.

2. Pfammatter JP, Gertsch M, Weber JW, Stocker FP, Moser H, Kappenberger L. Stress-induced polymorphous ventricular tachyarrhythmias in two brothers: unusual pattern of inheritance in the long QT syndrome. Clin Cardiol. 1993; 16(6):517-20.

3. Telles PD, Machado MA, Sakai VT, Nor JE. Pulp tissue from primary teeth: new source of stem cells. J Appl Oral Sci. 2011;19(3):189-94.

4. Nishino Y, Yamada Y, Ebisawa K, Nakamura S, Okabe K, Umemura E, et al. Stem cells from human exfoliated deciduous teeth (SHED) enhance wound healing and the possibility of novel cell therapy. Cytotherapy. 2011;13(5): 598-605. doi:10.3109/14653249.2010.542462.

5. Miura M, Gronthos S, Zhao M, Lu B, Fisher LW, Robey PG, et al. SHED: stem cells from human exfoliated deciduous teeth. Proc Natl Acad Sci U S A. 2003;100(10):5807-12. doi:10.1073/pnas.0937635100.

6. Parchment RE, Natarajan K. A free-radical hypothesis for the instability and evolution of genotype and phenotype in vitro. Cytotechnology. 1992;10(2):93-124.

7. Gudjonsson T, Villadsen R, Ronnov-Jessen L, Petersen OW. Immortalization protocols used in cell culture models of human breast morphogenesis. Cell Mol Life Sci. 2004;61(19-20):2523-34. doi:10.1007/s00018-004-4167-z.

8. Russo J, Tahin Q, Lareef MH, Hu YF, Russo IH. Neoplastic transformation of human breast epithelial cells by estrogens and chemical carcinogens. Environ Mol Mutagen. 2002;39(2-3):254-63. doi:10.1002/em.10052.

9. Band V. Preneoplastic transformation of human mammary epithelial cells. Semin Cancer Biol. 1995;6(3):185-92. doi:10.1006/scbi.1995.0015.

10. Wazer $\mathrm{DE}$, Chu Q, Liu XL, Gao Q, Safaii H, Band V. Loss of p53 protein during radiation transformation of primary human mammary epithelial cells. Mol Cell Biol. 1994;14(4):2468-78.

11. Band V. In vitro models of early neoplastic transformation of human mammary epithelial cells. Methods Mol Biol. 2003;223:237-48. doi:10.1385/1-59259-329-1:237.

12. DiPaolo JA. Relative difficulties in transforming human and animal cells in vitro. J Natl Cancer Inst. 1983;70(1):3-8.

13. Shay JW, Van Der Haegen BA, Ying Y, Wright WE. The frequency of immortalization of human fibroblasts and mammary epithelial cells transfected with SV40 large T-antigen. Exp Cell Res. 1993;209(1):45-52. doi:10.1006/excr.1993.1283.

14. Bryan TM, Reddel RR. SV40-induced immortalization of human cells. Crit Rev Oncog. 1994;5(4):331-57.

15. Dimova S, Brewster ME, Noppe M, Jorissen M, Augustijns P. The use of human nasal in vitro cell systems during drug discovery and development. Toxicol In Vitro. 2005;19(1):107-22. doi:10.1016/j.tiv.2004.07.003.

16. Egbuniwe O, Idowu BD, Funes JM, Grant AD, Renton T, Di Silvio L. P16/p53 expression and telomerase activity in immortalized human dental pulp cells. Cell Cycle. 2011;10(22):3912-9. doi:10.4161/cc.10.22.18093.

17. vom Brocke J, Schmeiser HH, Reinbold M, Hollstein M. MEF immortalization to investigate the ins and outs of mutagenesis. Carcinogenesis. 2006;27(11): 2141-7. doi:10.1093/carcin/bgl101.

18. Calado RT, Young NS. Telomere diseases. N Engl J Med. 2009;361 (24):2353-65. doi:10.1056/NEJMra0903373.

19. Bayne S, Jones ME, Li H, Pinto AR, Simpson ER, Liu JP. Estrogen deficiency leads to telomerase inhibition, telomere shortening and reduced cell proliferation in the adrenal gland of mice. Cell Res. 2008;18(11):1141-50. doi:10.1038/cr.2008.291.

20. Borssen M, Cullman I, Noren-Nystrom U, Sundstrom C, Porwit A,

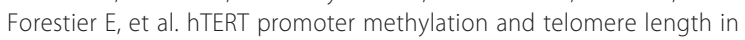
childhood acute lymphoblastic leukemia: associations with immunophenotype and cytogenetic subgroup. Exp Hematol. 2011;39(12):1144-51. doi:10.1016/.jexphem.2011.08.014

21. Venturini L, Daidone MG, Motta R, Collini P, Spreafico F, Terenziani M, et al. Telomere maintenance in Wilms tumors: first evidence for the presence of alternative lengthening of telomeres mechanism. Genes Chromosomes Cancer. 2011;50(10):823-9. doi:10.1002/gcc.20903.

22. Soares J, Lowe MM, Jarstfer MB. The catalytic subunit of human telomerase is a unique caspase-6 and caspase-7 substrate. Biochemistry. 2011;50(42): 9046-55. doi:10.1021/bi2010398.

23. Hao LY, Armanios M, Strong MA, Karim B, Feldser DM, Huso D, et al. Short telomeres, even in the presence of telomerase, limit tissue renewal capacity. Cell. 2005;123(6):1121-31. doi:10.1016/j.cell.2005.11.020.

24. Cohen $\mathrm{S}$, Jacob E, Manor H. Effects of single-stranded DNA binding proteins on primer extension by telomerase. Biochim Biophys Acta. 2004;1679(2): 129-40. doi:10.1016/j.bbaexp.2004.06.002.

25. Bianchi A, Shore D. How telomerase reaches its end: mechanism of telomerase regulation by the telomeric complex. Mol Cell. 2008;31(2):153-65. doi:10.1016/j.molcel.2008.06.013. 
26. Burns JS, Abdallah BM, Guldberg P, Rygaard J, Schroder HD, Kassem M. Tumorigenic heterogeneity in cancer stem cells evolved from long-term cultures of telomerase-immortalized human mesenchymal stem cells. Cancer Res. 2005;65(8):3126-35. doi:10.1158/0008-5472.CAN-04-2218.

27. Li N, Yang R, Zhang W, Dorfman H, Rao P, Gorlick R. Genetically transforming human mesenchymal stem cells to sarcomas: changes in cellular phenotype and multilineage differentiation potential. Cancer. 2009;115(20):4795-806. doi:10.1002/cncr.24519.

28. Huang G, Zheng Q, Sun J, Guo C, Yang J, Chen R, et al. Stabilization of cellular properties and differentiation multipotential of human mesenchymal stem cells transduced with hTERT gene in a long-term culture. J Cell Biochem. 2008;103(4):1256-69. doi:10.1002/jcb.21502.

29. Kelland LR. Overcoming the immortality of tumour cells by telomere and telomerase based cancer therapeutics - current status and future prospects. Eur J Cancer. 2005:41(7):971-9. doi:10.1016/j.ejca.2004.11.024.

30. Bocker W, Rossmann O, Docheva D, Malterer G, Mutschler W, Schieker M. Quantitative polymerase chain reaction as a reliable method to determine functional lentiviral titer after ex vivo gene transfer in human mesenchymal stem cells. J Gene Med. 2007:9(7):585-95. doi:10.1002/jgm.1049.

31. Vorsanova SG, Yurov YB, lourov IY. Human interphase chromosomes: a review of available molecular cytogenetic technologies. Mol Cytogenet. 2010;3:1. doi:10.1186/1755-8166-3-1.

32. Brothman AR, Persons DL, Shaffer LG. Nomenclature evolution: changes in the ISCN from the 2005 to the 2009 edition. Cytogenet Genome Res. 2009; 127(1):1-4. doi:10.1159/000279442.

33. Magaye R, Zhou Q, Bowman L, Zou B, Mao G, Xu J, et al. Metallic nickel nanoparticles may exhibit higher carcinogenic potential than fine particles in JB6 cells. PLoS One. 2014;9(4):e92418. doi:10.1371/journal.pone.0092418.

34. Bocker W, Yin Z, Drosse I, Haasters F, Rossmann O, Wierer M, et al. Introducing a single-cell-derived human mesenchymal stem cell line expressing hTERT after lentiviral gene transfer. J Cell Mol Med. 2008;12(4):1347-59. doi:10.1111/j.1582-4934.2008.00299.x.

35. Serakinci N, Guldberg P, Burns JS, Abdallah B, Schrodder H, Jensen T, et al. Adult human mesenchymal stem cell as a target for neoplastic transformation. Oncogene. 2004;23(29):5095-8. doi:10.1038/sj.onc.1207651.

36. Neoptolemos JP, Stocken DD, Bassi C, Ghaneh P, Cunningham D, Goldstein $D$, et al. Adjuvant chemotherapy with fluorouracil plus folinic acid vs gemcitabine following pancreatic cancer resection: a randomized controlled trial. JAMA. 2010;304(10):1073-81. doi:10.1001/jama.2010.1275.

37. Le Blanc K, Ringden O. Immunomodulation by mesenchymal stem cells and clinical experience. J Intern Med. 2007;262(5):509-25. doi:10.1111/j.1365-2796. 2007.01844.x.

38. Mimeault M, Batra SK. Recent insights into the molecular mechanisms involved in aging and the malignant transformation of adult stem/progenitor cells and their therapeutic implications. Ageing Res Rev. 2009;8(2):94-112. doi:10.1016/j.arr.2008.12.001

39. Herbig U, Jobling WA, Chen BP, Chen DJ, Sedivy JM. Telomere shortening triggers senescence of human cells through a pathway involving ATM, p53, and p21(CIP1), but not p16(INK4a). Mol Cell. 2004;14(4):501-13.

40. Simonsen JL, Rosada C, Serakinci N, Justesen J, Stenderup K, Rattan SI, et al. Telomerase expression extends the proliferative life-span and maintains the osteogenic potential of human bone marrow stromal cells. Nat Biotechnol. 2002;20(6):592-6. doi:10.1038/nbt0602-592.

41. Jun ES, Lee TH, Cho HH, Suh SY, Jung JS. Expression of telomerase extends longevity and enhances differentiation in human adipose tissue-derived stromal cells. Cell Physiol Biochem. 2004;14(4-6):261-8. doi:10.1159/000080335.

42. Shi S, Gronthos S, Chen S, Reddi A, Counter CM, Robey PG, et al. Bone formation by human postnatal bone marrow stromal stem cells is enhanced by telomerase expression. Nat Biotechnol. 2002;20(6):587-91. doi:10.1038/nbt0602-587.

43. Xu C, Jiang J, Sottile V, McWhir J, Lebkowski J, Carpenter MK. Immortalized fibroblast-like cells derived from human embryonic stem cells support undifferentiated cell growth. Stem Cells. 2004;22(6):972-80. doi:10.1634/stemcells.22-6-972.

44. Abdallah BM, Haack-Sorensen M, Burns JS, Elsnab B, Jakob F, Hokland P, et al. Maintenance of differentiation potential of human bone marrow mesenchymal stem cells immortalized by human telomerase reverse transcriptase gene despite [corrected] extensive proliferation. Biochem Biophys Res Commun. 2005;326(3):527-38. doi:10.1016/j.bbrc.2004.11.059.

45. Christiansen M, Kveiborg M, Kassem M, Clark BF, Rattan SI. CBFA1 and topoisomerase I mRNA levels decline during cellular aging of human trabecular osteoblasts. J Gerontol A Biol Sci Med Sci. 2000;55(4):B194-200.
46. Liu L, DiGirolamo CM, Navarro PA, Blasco MA, Keefe DL. Telomerase deficiency impairs differentiation of mesenchymal stem cells. Exp Cell Res. 2004;294(1):1-8. doi:10.1016/j.yexcr.2003.10.031.

47. Levine BL, Humeau LM, Boyer J, MacGregor RR, Rebello T, Lu X, et al. Gene transfer in humans using a conditionally replicating lentiviral vector. Proc Natl Acad Sci U S A. 2006;103(46):17372-7. doi:10.1073/pnas.0608138103.

48. Artandi SE, Chang S, Lee SL, Alson S, Gottlieb GJ, Chin L, et al. Telomere dysfunction promotes non-reciprocal translocations and epithelial cancers in mice. Nature. 2000:406(6796):641-5. doi:10.1038/35020592.

49. Willeit P, Willeit J, Mayr A, Weger S, Oberhollenzer F, Brandstatter A, et al. Telomere length and risk of incident cancer and cancer mortality. JAMA. 2010;304(1):69-75. doi:10.1001/jama.2010.897.

50. Weischer M, Nordestgaard BG, Cawthon RM, Freiberg JJ, Tybjaerg-Hansen A, Bojesen SE. Short telomere length, cancer survival, and cancer risk in 47102 individuals. J Natl Cancer Inst. 2013;105(7):459-68. doi:10.1093/jnci/djt016.

51. Kinzebach S, Bieback K. Expansion of mesenchymal stem/stromal cells under xenogenic-free culture conditions. Adv Biochem Eng Biotechnol. 2013;129:33-57. doi:10.1007/10_2012_134.

52. Haque N, Kasim NH, Rahman MT. Optimization of pre-transplantation conditions to enhance the efficacy of mesenchymal stem cells. Int J Biol Sci. 2015;11(3):324-34. doi:10.7150/ijbs.10567.

\section{Submit your next manuscript to BioMed Central and we will help you at every step:}

- We accept pre-submission inquiries

- Our selector tool helps you to find the most relevant journal

- We provide round the clock customer support

- Convenient online submission

- Thorough peer review

- Inclusion in PubMed and all major indexing services

- Maximum visibility for your research

Submit your manuscript at www.biomedcentral.com/submit
Ciomed Central 